\title{
Columbia digital news project: an environment for briefing and search over multimedia information
}

\author{
Alfred V. Aho ${ }^{1}$, Shih-Fu Chang ${ }^{2}$, Kathleen R. McKeown ${ }^{1}$, Dragomir R. Radev $^{1}$, John R. Smith ${ }^{2}$, Kazi A. Zaman \\ ${ }^{1}$ Department of Computer Science and New Media Technology Center, Columbia University, 1214 Amsterdam Ave., \\ NY 10027-7003, USA \\ ${ }^{2}$ Department of Electrical Engineering and New Media Technology Center, Columbia University, NY 10027, USA
}

\begin{abstract}
In this paper we describe an ongoing research project called the Columbia Digital News Project. The goal of this project is to develop a suite of effective interoperable tools with which people can find relevant information (text, images, video, and structured documents) from distributed sources and track it over a period of time. Our initial focus is on the development of a system with which researchers, journalists, and students can keep track of current news events in specific areas.
\end{abstract}

Keywords: Multimedia - News Delivery - Text Summarization - Image and Video Searching and Classification - Tracking Document Changes

\section{Introduction}

We report on an ongoing project that involves the development of technologies to aid people in finding and tracking the information they need to keep current in their jobs and lives. We are developing a system, the Columbia Digital News System (CDNS), that provides up-to-the-minute briefings on news of interest, linking the user into an integrated collection of related multimedia documents. Depending on the user's profile or query, events can be tracked over time with a summary given of the most recent developments. A representative set of images or videos can be incorporated into the summary. The user can follow up with multimedia queries to obtain more details and further information.

Our research is directed at producing a collection of interoperable multimedia tools with which users can manage knowledge, search for and track events, and summarize and present information. Our focus is on the development of efficient algorithms, modular components, and effective presentation methods. In this paper we outline our system architecture and describe the salient components of our system.

Correspondence to: A. Aho (aho@cs.columbia.edu)

\section{System architecture}

The architecture of the system that we are developing is shown in Fig. 1. Using a combination of textual keywords and visual features, the user can specify the type of information he or she is interested in tracking. The eventsearching and tracking module will look for matching information on the requested topics to find relevant text, images, and multimedia documents. The documents thus retrieved can be then sent to the summarizer and the results can then be presented and viewed.

The summary information extracted from the documents can be stored in database templates. In addition, the user may search online for related databases (e.g., in the current news domain, the CIA World Fact Book [4] contains relevant information) from which additional information can be gleaned and merged with information from the set of articles for the summary. At the same time, representative images and videos illustrating new information or common content can be selected to be included in the summary. On seeing the summary and related images or video, the user can request to see additional information or specific sources of interest. This is accomplished through a viewing and manipulation component that may reinvoke multimedia search using image and/or textual features.

Our system also contains tools for knowledge management. The novel aspect of these tools is their ability to collect, categorize, and classify image and video information and associated documents.

Several screens from a scenario of interaction with our existing system are shown below in Fig. 2. After providing a few keywords specifying the area of interest, the user can produce a summary of several articles on the World Trade Center bombing, including two representative images revealing what maps of the area and what photographs of the event are available. Currently, summarization is done using templates representing information extracted from the news article using a system developed for the DARPA message understanding conference (MUC) [7]. Since the MUC systems only handle South American terrorist news, we also store some hand- 


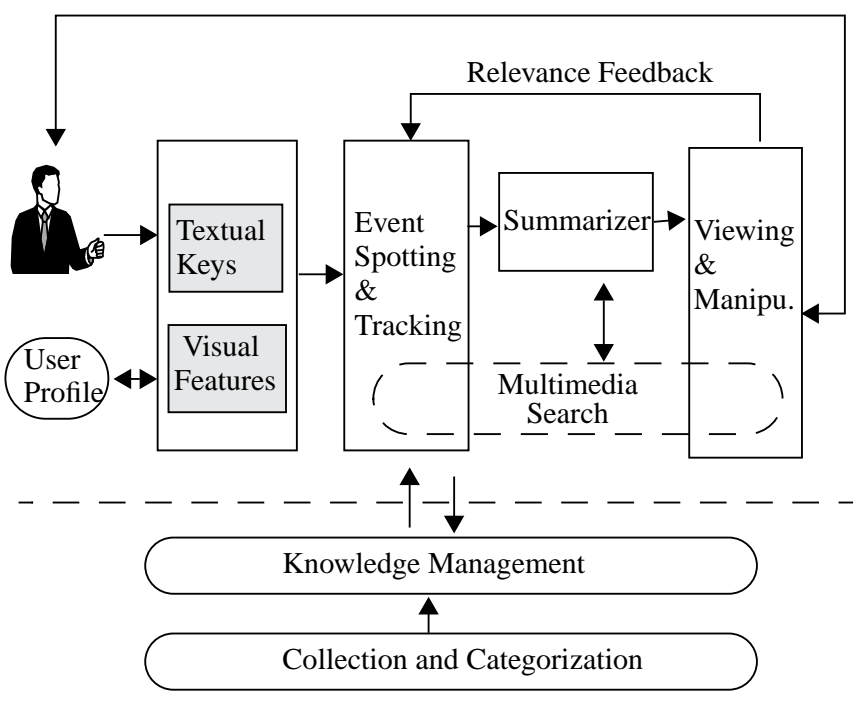

Fig. 1. System architecture

constructed templates in the same format for testing purposes. Following receipt of the summary, the user then can search for additional images, here choosing to search for images similar to the map. The image/video search engine uses content-based techniques to retrieve images with similar visual content $[2,18]$ (e.g., color and spatial structure in this case). This results in a set of 30 most relevant images (not shown) retrieved from our catalog of approximately 48000 images. The user then refines the image search by adding in textual keywords to specify the topic, resulting in the 3 images shown in the second screen of Fig. 2. Finally, the user can request additional textual documents relating to one of the retrieved images, receiving again a summary of the new documents retrieved.

Our current implementation is an early prototype illustrating our vision and therefore many open research issues remain. For example, we need to expand the kinds of media handled as well as interaction between media. By adding multimedia documents (news plus images), we can begin using the text within a document to aid in image searches. We can also use results from automated image subject classification to provide multimedia illustration in text summaries. In order to facilitate scaling the system, our work includes development of tools for building resources that can be used for searching, tracking, and summarization.

\section{Knowledge management}

The Columbia Digital News System contains a number of tools to help collect, categorize, and classify information. In order to track events of interest, CDNS must be able to identify new information that is related to the user's interests. We have tools for automatic information categorization to aid in this task as well as in improving the accuracy of any follow-up search and exploration that the user may wish to carry out. When a new image arrives, it can be automatically catalogued in the image taxonomy and the user can be notified if its category matches the user profile.

In addition to including an incoming image to the growing taxonomy, our work also features semi-automatic development of the image taxonomy using both image and textual features, active search of the World Wide Web to categorize images as part of our local collection, and use of the image taxonomy to improve the accuracy of follow-up search. While other groups have used image collections that were manually annotated with textual key words [8], our work focuses on semiautomatic cataloging through incremental classification into and extension of the taxonomy. This classification can then be subsequently used as an additional constraint on search to improve accuracy. In this section, we describe our current prototypes for image and video categorization.

\subsection{Cataloging images and video}

Aiming at a truly functional image/video search engine for online information, we have developed a tool called WebSeek [17] that issues a series of software agents (called spiders) to traverse the Web, automatically detect visual information, and collect it for automated cataloging and indexing. Taxonomies of knowledge are very useful for organizing, searching, and navigating through large collections of information. However, existing taxonomies are not well suited for handling dynamic online visual information such as images on the Web. We have developed a new working taxonomy for organizing image and video subject classes. Our WWW image/video cataloging system is unique in that it integrates both the visual features and text keys in visual material detection and classification. There are a few independently developed systems with similar goals $[6,8]$, but they do not use multimedia features to construct a comprehensive taxonomy like ours.

Our system uses both textual information and visual features to derive image/video type and subject classes. Type information indicates different forms of object content, such as "gif" and "jpeg" for images; "mpeg", "qt", and "avi" for video; and "htm" for html documents. Subject classes represent the semantic content of an image or video. They provide a valuable interpretation of the visual information, such as "astronomy/ planets" and "sports/soccer". We have developed fully and semi-automatic procedures for type/subject mapping.

We use two methods for image type mapping. The first method examines the type of the hyperlink and the filename extensions of the URLs. Mappings between filename extensions and object type are given by the MIME content type labels. This method provides reliable mappings when correct filename extensions are given. The second method is to use the visual features of the images/video for type assessment. This automated procedure involves training on samples of the color histograms of images and videos. Fisher discriminant analysis 


\section{QUERY OUTPUT}
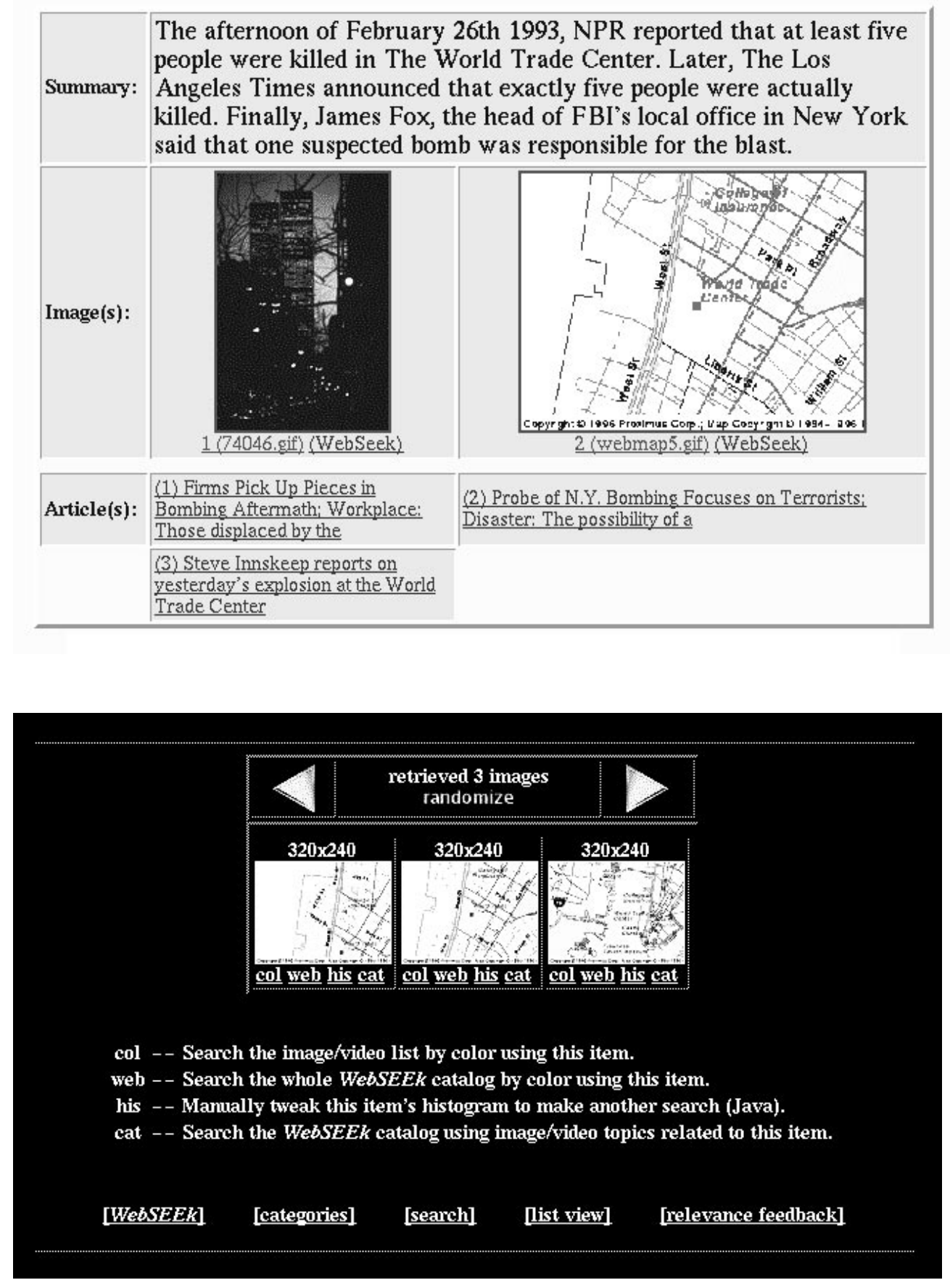

Fig. 2. a Illustrated summary generated by prototype $\mathbf{b}$ results of image search using both visual features and textual keys

is used to construct uncorrelated linear weightings of the color histograms that provide for maximum separation between training classes [17].

Mapping subject classes involves building an image/ video subject taxonomy. Our current taxonomy is built incrementally through the process of inspecting the keyterms associated with the visual information. For example, when new, descriptive terms such as "basketball" are automatically detected, a corresponding subject class is manually added to the taxonomy if it does not already exist, i.e., "sports/basketball".

Subject mapping for new image and video information involves a sequence of steps. First, key-terms are extracted automatically by examining textual information from hyperlinks (URLs and hyperlink tag) and directory names. Then, mapping between key-terms and the corresponding subject classes is done by using a keyterm dictionary, which is derived in a semi-automatic 
process. Based on the term histogram, terms with high frequency are presented for manual assessment. Terms are grouped into "descriptive" and "non-descriptive" classes. Only descriptive terms, such as "aircraft" and "texture" are added to the key-term dictionary. New images and videos associated with key-terms in the dictionary are automatically mapped to the corresponding subject classes.

\section{Searching and tracking}

Two significant features of CDNS are its facilities for searching for visual and textual information and its capabilities for tracking changes and related information. The multimedia search facility in CDNS allows users to find specific information by specifying query keys including both textual and visual properties. The tracking modules allow a user to produce an initial set of multimedia information relevant to his or her interest. The summarizer can then be invoked to produce briefings, possibly containing representative images for illustration. Scanning these multimedia briefings, users can examine the source documents and pursue an active process of in-depth information exploration.

\subsection{Content-based visual query and search}

Content-based visual query (CBVQ) techniques provide for the automated assessment of salient visual features such as color, texture, shape, motion, spatial, and temporal information contained within visual scenes [14]. By computing the similarities between images and videos using these extracted visual features, new powerful functionalities are added to the system:

- query based on features of visual information

- classification of images and video into meaningful semantic classes

- browsing and navigation by content through image and video archives [18]

- automated grouping of visual scenes into visually homogeneous clusters [20]

CBVQ brings about new technical challenges. Content analysis and feature extraction need to be fast for real-time database processing. Multiple features, including those from other media (e.g., text, audio), should be explored for object detection and indexing. Relevance feedback from user interaction is useful for adaptively choosing optimal features for different domains.

With these in mind, we are focusing on the following fundamental research issues in this area:

- fully automated extraction of visual features from both compressed and uncompressed images

- effective functions for measuring visual content similarity
- efficient indexing data structure enhancing fast feature matching

- fusion of multiple features and support of spatial queries

- linking low-level visual features to high-level semantics through intelligent clustering and learning

Our unique approach to processing compressed images is worth more extensive discussion. Due to their huge size, images and video are typically stored in compressed forms. A unique feature of our approach is the use of compressed data for feature extraction (such as localized color regions, texture regions, scenes, or video objects). Content-based queries are performed based on the extracted features. Uncompressed visual information is needed for display of final selected images or video only. The compressed-domain approach provides great benefits in reducing the resource requirements in computation, storage, and communication bandwidth.

Figure 3 shows the system architecture of a CBVQ system. Images stored in the archive are processed with automated content analysis tools to extract the prominent image regions with coherent features. The input query (either drawing or example image) is processed on the fly to obtain descriptions of its prominent regions. The associated visual features and their spatial information are compared against the image regions and features in the archive to find the matched results.

We have developed efficient algorithms and several CBVQ prototypes. Our early tool, VisualSEEk [18], is a fully automated image query system which supports both localized content query and spatial query. It allows users to specify visual features using a Java graphic interface. Users may specify arbitrarily-shaped regions with different features (e.g., color and texture) and their spatial relationship. Images matching the specified content are returned to users for viewing or further manipulation. The Java user interface for VisualSEEk is shown in Fig. 4.

An example of visual query using VisualSEEk is as follows. A user wants to find all images containing sunsets in the archive. By specifying a golden yellow circle in the foreground and a light brown rectangle in the background, he finds several sunset images within just seconds. The matches in terms of color regions and their spatial layout can be exact or best. From the returned images, he may choose one or more and ask the system to return other images similar to the selected one(s). Through multiple iterations of these two types of queries (query by example vs. query by sketch), the user can navigate through the entire collection. Relevance feedback is provided through the selection of relevant images in the returned batch.

The content-based visual search technique has been applied to the video domain as well. In VideoQ, users can search videos by specifying the animated sketches resembling the spatio-temporal attributes of video objects [1].

In a follow-up more advanced CBVQ tool called WebSEEk, we have integrated the search methods to 


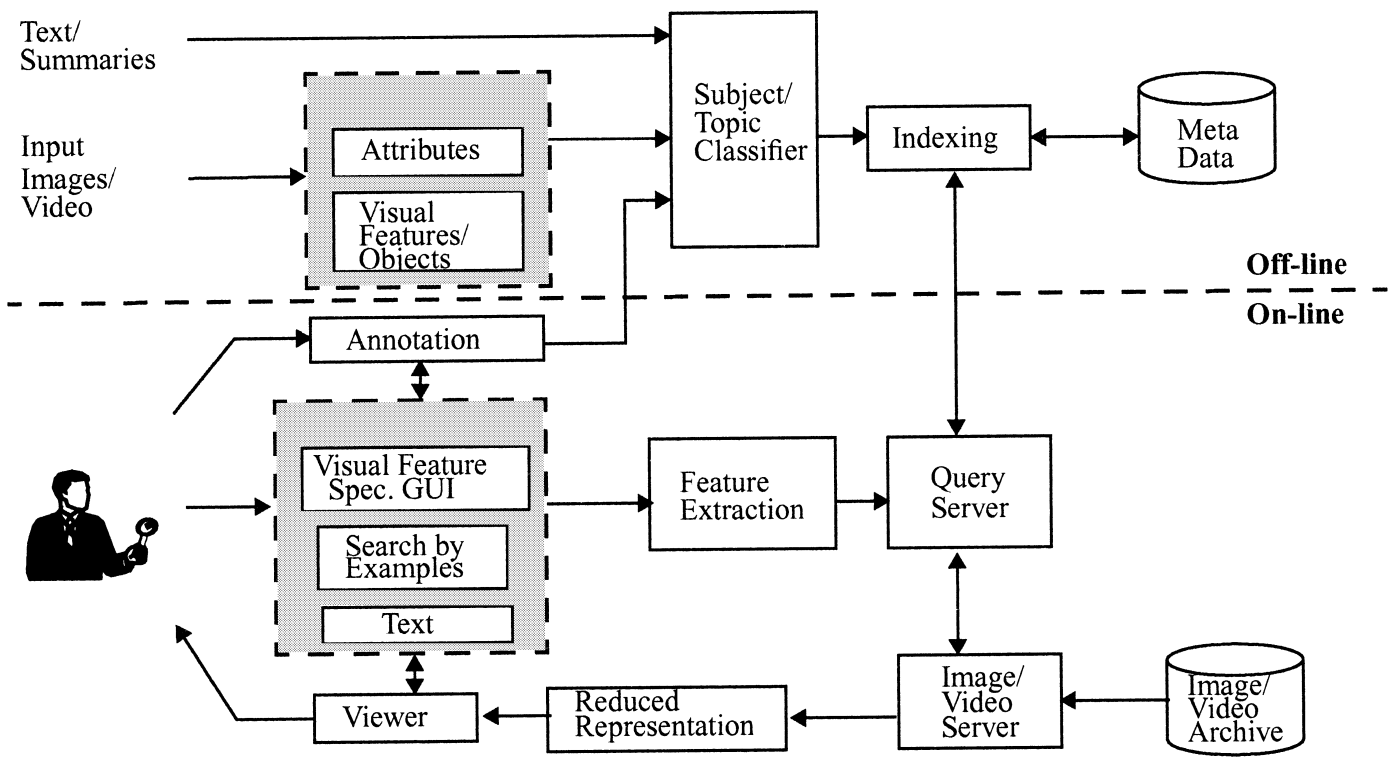

Fig. 3. CBVQ architecture

encompass both visual features and text keys. To search for images and videos, the user issues a query by entering terms or selecting subjects directly. The tool then extracts matching items from the catalog.

Following the initial search results, the user may perform follow-up search using tools integrating featurebased techniques and text-based tools.

\subsection{Tracking changes}

Changes and follow-ons to a document may be of even more interest than the original document itself. A news story, for example, may have subsequent follow-up stories containing additional information (e.g., suspect was now caught) or corrections to previous stories (e.g., additional victims were found). Ideally, the user would like to track the changes in an ongoing story and have the results reported in a meaningful and understandable way.

Our approach to this problem is to develop tools that identify and track changes in multimedia documents. The changes can then be fed into the summarization modules for presentation to the user. Since our current application is journalism, we are investigating difference measures that work well on news stories.

Tracking changes in news stories can be broadly divided into two parts. The first problem is that of collection. Here we have multiple sources of information and we have to determine which documents would be of interest to the user. The second problem is that of developing a suitable internal representation for the various threads in the story and representing the relationships between them.

The collection problem is similar to that of the document routing problem. We use structural techniques to detect the presence of new stories on a web page and use a classifier to determine the appropriate position of this story in our internal representation. The various threads in this internal representation serves as input to the classifier.

For structural differences, we are building on recent work done at Stanford [3] for measuring change detection in hierarchies of objects. The structural properties we employ are based on the grammatical structure of an HTML page as well as other kinds of metadata associated with a document. Although we describe the specific problem of dealing with HTML pages, it should be noted that our techniques are applicable to other domains and formats as well.

We use statistical natural language processing techniques to focus on semantic differences between two documents. In information retrieval, various vectorbased techniques have been developed to estimate the similarity of the content of documents. Since news stories are typically short in length, and therefore in pairs do not provide enough data for training, we find simpler metrics preferable. We characterize a document by its content words, that is, by its nouns, adjectives, and verbs; other parts of speech, such as articles, adverbs, and prepositions, are ignored. We use a part-of-speech tagger to identify the content words in the documents under consideration. The statistical metrics we currently use are based on the number of content words common to the documents. The content words which characterize a document are the basis of the interdocument relationships. The interdocument relationships provide the user with a temporal flow of the news story as well as a semantic flow. If the story under consideration is the death of Princess Diana, the threads could diverge into directions as different as celebrity car crashes and the future of the monarchy. 


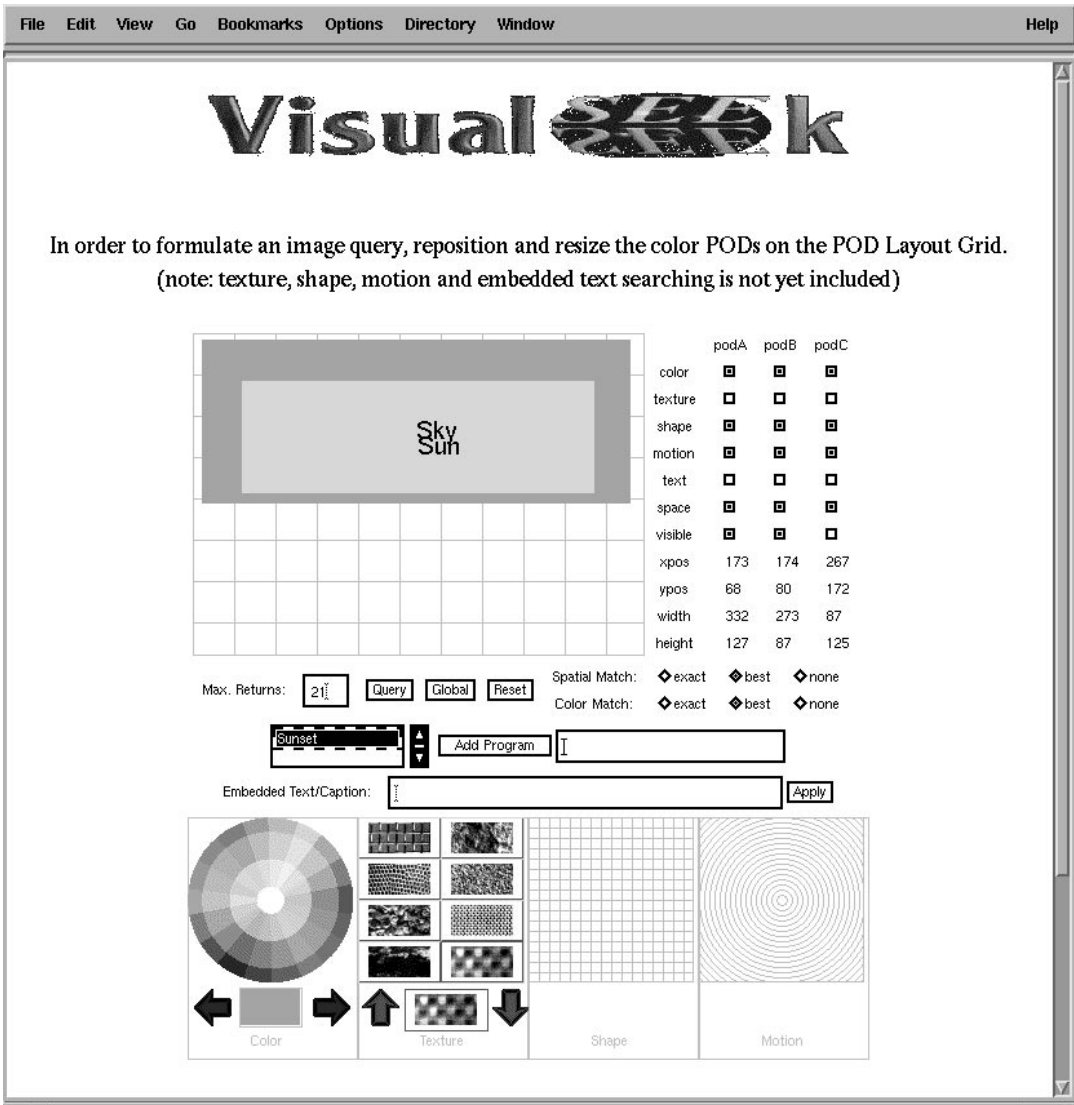

Fig. 4. VisualSEEk user interface: a users specify color regions to find sunset images $\mathbf{b}$ results 


\section{Summarization}

The goal of summarization within CDNS is to brief the user on information within the documents retrieved by tracking. In many cases, the summary may provide enough information for the user to skip reading the original document. In others, the user may want to check the original documents to verify information contained within the summary, to follow up on an item of interest, or to resolve conflicting information between sources. Summary generation is strongly shaped by the characteristics of the tracking environment. At any particular point in time, the summarization component will be given a set of documents on the same event from a specific time period and at later points, will receive additional later breaking news on the same event. To meet the demands of this environment, our work on summarization includes the following key features:

- Summaries are generated over multiple articles, merging information from all relevant sources into a concise statement of the facts. This is in contrast to most previous work that summarizes single articles [9, $15,11]$.

- Summaries must identify how perception of the event changes over time, distinguishing between accounts of the event and the event itself [13].

- Given access to live news, the summarizer must provide an update since the last generated summary, identifying new information and linking its presentation to earlier summaries.

Given the multimedia nature of the tracking environment, presentation of tracked information must also include multimedia information. We make use of our categorization tools to select a representative sample of retrieved images that are relevant to, or part of, the textual news. If images are an item of interest, journalists can follow up on the summary by requesting images similar to one or more of the representative images.

In the remainder of this section, we present the main phases of summary generation in CDNS. Unlike previous work on summarization, CDNS does not extract sentences from the input articles to serve as the summary. Instead, we use natural language processing techniques to extract structured information from the document and language generation techniques [12] to merge information extracted from different documents and form the language of the summary. Our research focus is on problems in the language generation stage. In the following sections, we describe the tools we have developed and use for information extraction, selection of summary content (conceptual summarization), and determination of summary wording and phrasing (linguistic summarization), all embodied in our prototype system, SUMMONS [13]. The architecture of SUMMONS is shown in Fig. 5.

\subsection{Information extraction}

Information extraction tools are used to find and extract information of interest from the set of input articles and represent them in a template format. In particular, we use existing systems for extracting information specific to terrorist events and have developed a tool for extracting names and their descriptions, and tools for connecting into online structured data.

Currently we are using an information extraction system for the domain of terrorist news articles developed under the DARPA human language technology program, NYU's Proteus system [7]. Proteus filters irrelevant sentences from the input articles, and uses parsing and pattern matching techniques to identify event type, location, perpetrator, and victim, among others, building a template representation of the event as shown in Table 1. The template representations for the set of articles are passed as input to the summary generation component. Coverage of Proteus is limited to South American terrorist activities. In order to handle common events in current news (e.g., terrorism in the Mid East or the US), we are enhancing this approach by developing new patterns and implementing them as part of another information extraction system, CIRCUs system, developed at the University of Massachusetts [10], which provides for representation and semi-automatic acquisition of new patterns.

We have also developed separate pattern matching techniques to extract specific types of information from input text, building domain knowledge sources for use in text generation. We have built a prototype tool, called PROFILE, which uses news corpora available on the Internet to identify company and organization names, person names along with descriptions (e.g., South Africa's main black opposition leader, Mangosuthu Buthelezi or Sinn Fein, the political arm of the Irish Republican Army), building a large-scale lexicon and knowledge source. Descriptions of entities are collected over a period of time to ensure large lexical coverage. Our database contains automatically retrieved descriptions of 12453

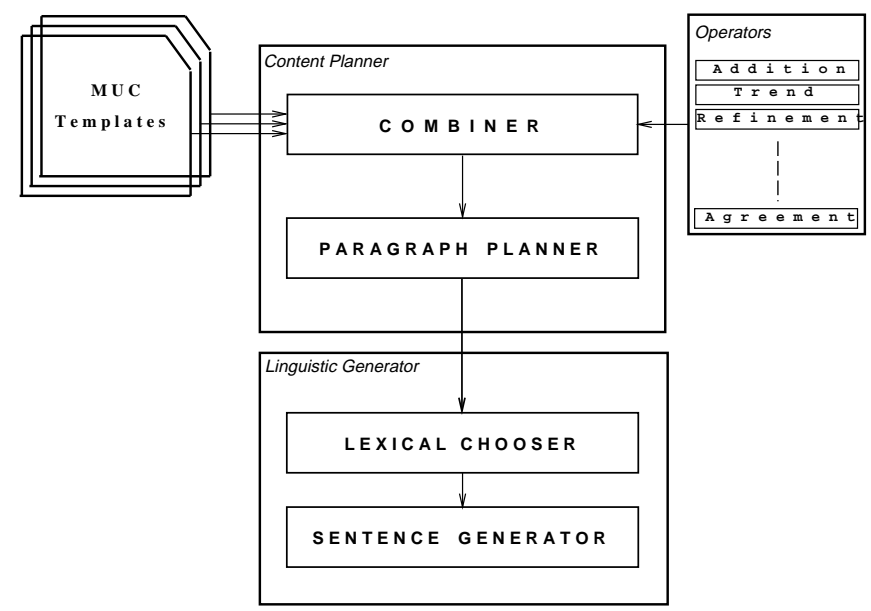

Fig. 5. SUMMONS system architecture 
Table 1. Portions of a sample template

\begin{tabular}{rll}
\hline 0. & MESSAGE: ID & TST2-MUC4-0048 \\
1. & MESSAGE: TEMPLATE & 1 \\
2. & INCIDENT: DATE & 19 APR 89 \\
3. & INCIDENT: LOCATION & EL SALVADOR: SAN SALVADOR (CITY) \\
4. & INCIDENT: TYPE & ATTACK \\
5. & INCIDENT: STAGE OF EXECUTION & ACCOMPLISHED \\
8. & PERP: INCIDENT CATEGORY & TERRORIST ACT \\
10. & PERP: ORGANIZATION ID & "FMLN" \\
11. & PERP: ORGANIZATION CONFIDENCE & SUSPECTED OR ACCUSED: "FMLN" \\
18. & HUM TGT: NAME & "ROBERTO GARCIA ALVARADO" \\
19. & HUM TGT: DESCRIPTION & "ATTORNEY" \\
& & "ATTORNEY": "ROBERTO GARCIA \\
20. & HUM TGT: TYPE & ALVARADO" \\
& & ACTIVE MILITARY: “ATTORNEY", \\
21. & HUM TGT: NUMBER & LEGAL OR JUDICIAL: "ROBERTO \\
23. & HUM TGT: EFFECT OF INCIDENT & GARCIA ALVARADO" "ATTORNEY" \\
& & 1: "ROBERTO GARCIA ALVARADO", \\
\hline
\end{tabular}

entities at this moment. CDNS also includes tools for access to structured online databases, and currently can retrieve information from the CIA Fact Book [4] and the George Mason database of terrorist organizations [19], both of which provide information relevant to terrorism and current affairs in general. Our next step will be to incorporate information extracted by PROFILE or from remote databases in a summary.

\subsection{Conceptual summarization}

Conceptual summarization requires determining which information from the set of possible extracted information to include in the summary, how to combine information from multiple sources, and how to organize it coherently.

Conceptual summarization uses planning operators, identified through corpus analysis, to combine information from separate templates into a single template. The summarizer includes seven planning operators, which identify differences and parallels between templates such as contradictions or agreement between sources, addition of new information, or refinement of existing information. Each planning operator is written as a rule, containing preconditions which must be met for the rule to apply, and actions which extract or modify information from the input templates creating a new template.

\subsection{Linguistic summarization}

Once the content of the summary is determined by the conceptual summarization components, it is represented as one or more templates created by merging the templates for each article. This representation is passed to the linguistic summarization components which determine the actual wording to be used in the summary. This requires first creating a case frame representation for each template, selecting which template field will be realized as the verb of the sentence, and mapping other fields to the arguments of the verb. During this process, words are selected to realize the values of the fields. The resulting case frame is passed through syntactic generation, where a full syntactic tree of the sentence is created, grammatical constraints are enforced, and morphological agreement is carried out. We use Columbia's FUF/ SURGE package [5, 16], a robust grammar of English (SURGE) along with a unification interpreter (FUF), and some text manipulation tools written in Perl as the basis for both word choice and syntactic generation.

\section{Conclusions and status}

In the CDNS project, we have developed tools and a system architecture with which users can manage knowledge, summarize information, and search for and track events. The current CDNS integrates an implemented, domain-specific summarization prototype and a largescale online domain-independent prototype for contentbased visual query and Web image/video search. In addition, we have developed robust tools for extraction of names and descriptions, for cataloging images based on multimedia features, and for evaluating methods for measuring differences in Web documents. Although the tools that we have developed can facilitate access to live documents, CDNS primarily works on locally stored documents. Our current efforts focus on enhancing access to live data, integrating more sophisticated use of multiple media in image classification, and extending our algorithms for detecting differences in multiple versions of documents to tracking series of changes in a story over time.

Currently, we are extending our system in three main directions: more sophisticated integrated of visual and text features for image classification, event tracking and search, and the generation of summary updates. In image 
classification, we plan to perform learning over the image taxonomy to derive mappings of feature clusters to semantic objects. We are also investigating how best to use the full text associated with images in multimedia documents to improve classification accuracy. In tracking and search, we are building a set of tools for automated creation of threads of related news articles. In our future work, we will also link our image search engines to video browsing/editing systems which will result in a more powerful environment for visual information access and manipulation. Finally, in news summarization, as we move to live news feeds, we must extend CDNS so that it can update a user on the latest breaking news, without unnecessarily including information provided earlier. At the same time, the summaries will include appropriate historical material which puts the new briefing in context.

Acknowledgement. This material is based upon work supported by the National Science Foundation under Grant No. IRI 96-19124 and by a grant from Columbia University's Strategic Initiative Fund. Work on summarization was also supported in part by the National Science Foundation under Grant No. GER 90-24069 and IRI 96-25374. Work on content-based visual query was also supported in part by the National Science Foundation under a CAREER award (IRI-95-01266), and industrial sponsors of Columbia's ADVENT projects. Any opinions, findings, and conclusions or recommendations expressed in this material are those of the authors and do not necessarily reflect the views of the National Science Foundation.

\section{References}

1. S.-F. Chang, W. Chen, H. J. Meng, H. Sundaram, D. Zhong. VideoQ - an automatic content-based video search system using visual cues. In: Proc. ACM Intern. Conf. Multimedia, Seattle, WA, November 1997. ACM. Demo accessible from URL http:// www.ctr.columbia.edu/videoq

2. S.-F. Chang, J. R. Smith, H. J. Meng, H. Wang, D. Zhong. Finding images/video in large archives-Columbia's content-based visual query projects. CNRI Digital Library Magazine, February 1997

3. S. Chawathe, S. Rajaraman, H. Garcia-Molina, J. Widom. Change detection in hierarchically structured information. In: Proc. ACM SIGMOD Symposium. ACM, 1996

4. CIA. The CIA World Factbook 1995. URL: http:// www.odci.gov/ cia/publications/95fact

5. Michael Elhadad. Using argumentation to control lexical choice: a functional unification implementation. PhD thesis, Department of Computer Science, Columbia University, New York, 1993

6. C. Frankel, M. Swain, V. Athitsos. WebSeer: An image search engine for the World Wide Web. Technical Report TR-96-14, University of Chicago, July 1996
7. Ralph Grishman, Catherine Macleod, John Sterling. New York University: Description of the PROTEUS system as used for MUC4. In: Proc. of the 4th Message Understanding Conference, June 1992

8. Interpix. Image Surfer. Technical Report http://www.interpix.com/

9. Julian M. Kupiec, Jan Pedersen, Francine Chen. A trainable document summarizer. In: Edward A. Fox, Peter Ingwersen, and Raya Fidel (eds.) Proc. of the 18th Annual International ACM SIGIR Conference on Research and Development in Information Retrieval, pp. 68-73, Seattle, WA, July 1995

10. Wendy Lehnert, Joe McCarthy, Stephen Soderland, Ellen Riloff, Claire Cardie, Jonathan Peterson, Fangfang Feng. UMass/Hughes: Description of the CIRCUS system used for MUC-5. In: Proc. of the 5th Message Understanding Conference (MUC-5), pp. 277-291, Baltimore, MD, August 1993

11. Hans P. Luhn. The automatic creation of literature abstracts. IBM Journal, pp. 159-165, 1958

12. Kathleen R. McKeown. Text generation: using discourse strategies and focus constraints to generate natural language text. Studies in Natural Language Processing. Cambridge University Press, Cambridge, UK, 1985

13. Kathleen R. McKeown, Dragomir R. Radev. Generating summaries of multiple news articles. In: Edward A. Fox, Peter Ingwersen, and Raya Fidel (eds.) Proc. of the 18th Annual International ACM SIGIR Conference on Research and Development in Information Retrieval, pp. 74-82, Seattle, WA, July 1995

14. W. Niblack, R. Barber, W. Equitz, M. Flickner, E. Glasman, D. Petkovic, P. Yanker, C. Faloutsos. The QBIC project: Querying images by content using color, texture, and shape. In: Storage and Retrieval for Image and Video Databases, SPIE Vol. 1908, February 1993

15. Lisa F. Rau, R. Brandow, K. Mitze. Domain-independent summarization of news. In: Summarizing Text for Intelligent Communication, pp. 71-75, Dagstuhl, Germany, 1994

16. Jacques Robin. Revision-based generation of natural language summaries providing historical background. PhD thesis, Department of Computer Science, Columbia University, New York, 1994

17. J. R. Smith, S.-F. Chang. Visually searching the web for content. IEEE multimedia magazine, July - September, 4: 3 (1997), pp. 1220

18. J. R. Smith, S.-F. Chang. VisualSEEk: a fully automated contentbased image query system. In: Proc. ACM Intern. Conf. Multimedia (ACMMM) Boston, MA, November 1996, pp. 87-98

19. The Terrorist Profile Weekly. The TPW terrorist group index 1996. URL: http://www.site.gmu.edu/ cdibona/grpindex.html

20. D. Zhong, H. Zhang, S.-F. Chang. Clustering methods for video browsing and annotation. In: IS\&T/SPIE Symposium on Electronic Imaging: Science and Technology - Storage \& Retrieval for Image and Video Databases IV, San Jose, CA, February 1996 